\title{
Non-invasive measures of coronary microcirculation: Taking the long road to the clinic
}

\author{
Kieran S. Chung, BS, ${ }^{\mathrm{a}}$ and Patricia K. Nguyen, $\mathrm{MD}^{\mathrm{a}, \mathrm{b}}$ \\ a Cardiology Section, Veterans Affairs Palo Alto Health Care Administration, Palo Alto, CA \\ b Division of Cardiovascular Medicine, Department of Medicine, Stanford University, Stanford, \\ CA
}

Received Jun 15, 2017; accepted Jun 16, 2017

doi: 10.1007/s12350-017-0972-2

\begin{abstract}
Although coronary microvascular disease is now a well-recognized entity that is associated with significant morbidity and mortality, current non-invasive strategies cannot differentiate between coronary microvascular disease (CMD) and obstructive epicardial stenosis. While the evaluation of intramyocardial blood volume as a surrogate measure for microvascular health may have limited sensitivity in early-stage disease, this strategy does enable the diagnosis of CMD in the presence of concurrent epicardial disease, bringing us one step further toward improving the management of this disease. Herein, we discuss the advantages and limitations of current non-invasive measures of CMD and the need for further investment in bringing these technologies to the bedside.
\end{abstract}

Key Words: Coronary microcirculation • myocardial perfusion imaging: SPECT • coronary artery disease $\cdot$ coronary blood flow $\bullet$ PET/CT imaging $\cdot$ diagnostic and prognostic application

\section{See related article, pp. 2096-2111}

Atherosclerotic plaque can develop in the large epicardial arteries, leading to the development of ischemic heart disease (IHD), a major cause of death worldwide. ${ }^{1}$ Although the diagnosis and treatment of epicardial coronary stenosis remain the primary focus of IHD management, patients with IHD may also have concurrent damage to the inner lining of the tiny coronary arteries that constitute the coronary microcirculation, resulting in spasm and decreased myocardial blood flow. ${ }^{2-4}$ Recent studies have shown that patients who have coronary microvascular disease (CMD) with and without significant obstructive epicardial disease are

\footnotetext{
Reprint requests: Patricia K. Nguyen, Division of Cardiovascular Medicine, Department of Medicine, Stanford University, 300 Pasteur Drive, Grant Building S114, CA 94301; pknguyen@stanford.edu

J Nucl Cardiol 2018;25:2112-5.

$1071-3581 / \$ 34.00$

Copyright @ 2017 American Society of Nuclear Cardiology.
}

at increased risk for cardiac-related morbidity and mortality. ${ }^{5,6}$ Due to the lack of robust and accurate noninvasive measures to evaluate CMD in the presence of concurrent epicardial stenosis, CMD remains an underdiagnosed and under-treated disease. ${ }^{7}$

Because of the need for exquisite spatial resolution to visualize this tiny complex vascular network, available invasive and non-invasive strategies cannot directly evaluate the human coronary microcirculation in vivo. Instead, these techniques indirectly measure parameters that are dependent on the functional integrity of the microcirculation. Several invasive (e.g., Dopplerderived coronary flow reserve and index of microcirculatory resistance) and non-invasive strategies (e.g., myocardial flow reserve by PET, Echo, and MRI) ${ }^{8-11}$ have been employed to study the coronary microcirculation. Compared to non-invasive strategies, the major limitations of invasive approaches include the following: (1) exposure to potential complications of invasive angiography, (2) limited ability to serially measure CMD for therapeutic monitoring, and (3) the need for sequential interrogation of each main coronary artery 
segment to provide a complete evaluation for CMD, which is rarely performed. Catheter-based measures, however, can account for the presence of concurrent epicardial stenosis, which can affect the coronary microcirculation and remain the major limitation for non-invasive approaches. Finally, most invasive and non-invasive approaches require the administration of a stress agent, which poses additional risk and limits throughput.

The work by Mohy-ud-Din et $\mathrm{al}^{12}$ addresses the need to find an accurate and reliable non-invasive index that can not only quantify the health of coronary microvasculature, but can also differentiate between CMD and significant epicardial stenosis. The investigators based their study on the presumption that the measurement of resting intramyocardial blood volume (IMBV) reflects the health of the microcirculation. The strength of this premise relies on findings from previous studies demonstrating that the primary site of auto-regulation occurs in the coronary microcirculation, ${ }^{13}$ which contributes to IMBV through arteriolar dilation and capillary recruitment. If microvascular function becomes impaired, auto-regulation will be adversely affected, thereby, blunting IMBV. This hypothesis was tested in a previous study by $\mathrm{Wu}$ et al, ${ }^{14}$ who measured changes in IMBV using computed tomography performed in dogs under controlled hemodynamic conditions at rest, in the presence and absence of significant epicardial stenosis, and after various degrees of microembolization. Although the study demonstrated that changes in IMBV under resting conditions paralleled changes in the microcirculation after embolization even in the presence of significant large vessel flow obstruction, a decrease in IMBV was only noted after $50 \%$ of the microcirculation was blocked, questioning the sensitivity of IMBV for detecting more modest decreases $(<50 \%)$ in the microcirculation. However, given the lack of non-invasive measures that can diagnose CMD in the presence of obstructive epicardial disease, using IMBV may be the best and only alternative.

The present study set out to ascertain the feasibility of accurately quantifying IMBV in vivo using ${ }^{99 m} \mathrm{Tc}$ RBC SPECT/CT imaging, in order to establish a noninvasive imaging methodology to assess myocardial microcirculatory function independent of co-existing epicardial disease. This strategy, however, can result in an overestimation IMBV. Because there is a higher RBC tracer concentration within the blood pool than the adjacent myocardium, spill-in activity from partial volume effect, scatter, and respiratory/cardiac motion can cause an increase in the calculated IMBV. The authors have addressed these technical limitations by implementing the following corrections to these physical and physiological degradation factors: (1) cardiac gating
(CG), (2) attenuation correction (AC), (3) scatter correction (SC), (4) dual cardiorespiratory gating (DG), and (5) partial volume correction (PVC). The authors approached the implementation of correction factors systematically by applying the following five combinations during end diastolic and end systolic acquisition phases: (1) CG, (2) $\mathrm{CG}+\mathrm{AC}$, (3) $\mathrm{CG}+\mathrm{AC}+\mathrm{SC}$, (4) $\mathrm{DG}+\mathrm{AC}+\mathrm{SC}$, (5) $\mathrm{DG}+\mathrm{AC}+\mathrm{SC}+\mathrm{PVC}$. IMBV estimates adjusted by each correction scheme were then compared to a designated gold standard using the Student's $t$ test, linear regression analysis, and quantitative agreement using Bland-Altman analysis.

In their study, 12 female beagle dogs $(10.8 \pm 1.0 \mathrm{~kg})$ were scanned using a Cadmium Zinc Telluride (CZT) detector. Six of the twelve canine hearts were used to validate the new IMBV quantification methodology through a comparison with ex vivo microCT imaging of the explanted casted hearts (e.g., "the gold standard',). IMBV was calculated as the fraction of ${ }^{99 \mathrm{~m}} \mathrm{Tc}-\mathrm{RBC}$ uptake in the myocardium over the tracer uptake in the left ventricular blood pool. In addition to resting IMBV, cycle-dependent change in IMBV was calculated as a percentage of the difference of IMBV during diastole and systole over the total IMBV during diastole. Although the IMBV ex vivo SPECT/CT measurements consistently overestimated the resting IMBV calculation made by the respective microCT images, IMBV measured by SPECT/ CT was highly correlated with IMBV measured by the microCT, especially using SPECT/CT images that were adjusted with the full correction scheme (e.g., DG + $\mathrm{AC}+\mathrm{SC}+\mathrm{PVC})$. Upon visual inspection, the full correction scheme provided images with a much higher resolution and improved functional boundaries, primarily due to the addition of a correction for partial volume effects using the proposed Yang approach. ${ }^{15}$ Bland-Altman analysis demonstrated that the full correction scheme significantly reduced the positive bias in the estimation of IMBV. In contrast to resting IMBV, cycledependent changes in IMBV were consistently underestimated compared to the microCT IMBV calculation. Again, Bland-Altman analysis demonstrated most agreement with the full correction scheme through successful mitigation of negative bias when applied to SPECT/CT IMBV. Based on these results, the authors have successfully demonstrated the feasibility of in vivo IMBV quantification using ${ }^{99 \mathrm{~m}} \mathrm{Tc}-\mathrm{RBC}$ SPECT/CT imaging.

Of note, the most significant improvement in the accuracy of IMBV quantification resulted from the addition of the PVC factor. ${ }^{16,17}$ PVC was performed using co-registered and phase-matched SPECT, contrast-enhanced CT, and attenuation mapping. The data were applied through the iterative Yang approach with high-resolution re-sliced ROI's of the myocardium, blood pool, lung, liver, and background, and with 
boundary tissue fraction thresholds. ${ }^{12,18}$ Ultimately, Mohy-ud-Din, et al's approach to image reconstruction evaluated IMBV using a single ROI to correct for partial volume effects over the entire myocardium. The authors hypothesized that total IMBV would be decreased in patients with microvascular dysfunction when compared to patients with healthy microcirculation as a result of microvascular rarefaction (e.g., reduced density of capillaries) involving the entire myocardium. ${ }^{19,20}$

Challenging the potential application of the authors' current strategy, recent literature has argued that IMBV distribution rather than total IMBV differs significantly between patients with and without CMD. ${ }^{21}$ In 2014, ${ }^{21}$ Behrenbeck et al published a case control study looking at early changes in myocardial microcirculation in asymptomatic patients with and without dyslipidemia ( $n=24$ per group) using CT myocardial perfusion. To overcome the challenge of inadequate spatial resolution of CT to resolve the microvasculature, the authors focused on the distribution of myocardial perfusion and IMBV, taking a regional blood flow approach to quantification. There were no significant differences between the two groups in overall myocardial perfusion and total IMBV. To assess regional distribution of IMBV, Behrenbeck and colleagues employed a sub-ROI scheme that they called a "nested ROI" (nROI) sampling methodology in addition to the total myocardium ROI. The nROI data showed a significant increase in spatial heterogeneity in the "at risk" group when compared to the control group. The increased spatial heterogeneity is hypothesized to be an indication of microvascular dysfunction. A subsequent study published by the same group in $2015,{ }^{22}$ using the same techniques evaluated early changes in myocardial microcirculation in patients with and without dilated cardiomyopathy ( $n=24$ per group). Similar to the results from their 2014 study, there was no difference between the two groups in overall myocardial perfusion and IMBV, but there were significantly differences in spatial heterogeneity across sub-ROI regions in the myocardium between patients with and without dilated cardiomyopathy. In the defense of Mohy-ud-Din et al, ${ }^{12}$ it is possible that total IMBV is not sensitive enough to detect early-stage disease, as suggested by previous studies, ${ }^{14}$ and, thus could not detect CMD in these asymptomatic patients. In the event that these patients were at the primary stage of disease, there may still be potential for total IMBV to serve as a valuable index for symptomatic patients with more severe CMD.

Although Behrenbeck et al, ${ }^{21,22}$ may have slightly different evidence at the moment regarding the value of IMBV quantification and its ability to represent microcirculatory health when compared to the method proposed by Mohy-ud-Din et al, they do agree that early changes in
IMBV can be used to glean valuable information regarding microcirculatory function. With limited evidence to support either total IMBV versus spatial distribution of IMBV as an index of microcirculatory health, the best way to move forward is to focus on accumulating strong scientific evidence. It would be interesting to see whether the technique proposed by Mohy-ud-Din et al can evaluate patients with varying degrees of CMD in the absence and presence of epicardial stenosis with good reproducibility and accuracy. Establishing resting IMBV and cycle-dependent changes in IMBV as trusted indexes of myocardial microcirculation would be an enormous step forward and would pave the way for evaluating its prognostic value for predicting adverse events as well as its ability to sequentially monitor patients to evaluate novel treatment strategies.

Despite the encouraging results from Mohy-ud-Din et al, we have a long road ahead before these techniques are used routinely in patients. Even established techniques like invasive angiography that measure coronary flow reserve or the index of microvascular resistance and non-invasive techniques that evaluate myocardial blood flow by PET, echo, and MRI are only performed at specialized centers or used mainly for research purposes, despite numerous studies establishing their utility in this population. The reasons for this underutilization are unclear. Perhaps most patients with suspected CMD have concurrent epicardial stenosis, limiting the diagnostic accuracy for non-invasive techniques. Alternatively, providers may choose a therapeutic trial of beta-blockers or nitrates in lieu of a diagnostic test because surgical intervention is not an option. Finally, there may be a lack of awareness of this entity among providers. Given strong evidence showing that CMD adversely affects cardiovascular morbidity and mortality, ${ }^{5,6}$ there should be greater impetus to develop more robust non-invasive imaging strategies in the near future that can routinely diagnose and manage patients with CMD in the clinical setting.

\section{Disclosure}

The authors declare that there is no conflict of interest to disclose.

\section{References}

1. Benjamin EJ, Blaha MJ, Chiuve SE, et al. Heart disease and stroke statistics-2017 update: A report from the American Heart Association. Circulation. 2017;135:e146-603.

2. Dean J, Cruz SD, Mehta PK, Merz CN. Coronary microvascular dysfunction: Sex-specific risk, diagnosis, and therapy. Nat Rev Cardiol. 2015;12:406-14. 
3. Petersen JW, Pepine CJ. Microvascular coronary dysfunction and ischemic heart disease: Where are we in 2014? Trends Cardiovasc Med. 2015;25:98-103.

4. Camici PG, Crea F. Coronary microvascular dysfunction. N Engl J Med. 2007;356:830-40.

5. Pepine CJ, Anderson RD, Sharaf BL, et al. Coronary microvascular reactivity to adenosine predicts adverse outcome in women evaluated for suspected ischemia results from the National Heart, Lung and Blood Institute WISE (Women's Ischemia Syndrome Evaluation) study. J Am Coll Cardiol. 2010;55:2825-32.

6. von Mering GO, Arant CB, Wessel TR, et al. Abnormal coronary vasomotion as a prognostic indicator of cardiovascular events in women: Results from the National Heart, Lung, and Blood Institute-Sponsored Women's Ischemia Syndrome Evaluation (WISE). Circulation. 2004;109:722-5.

7. Marinescu MA, Loffler AI, Ouellette M, Smith L, Kramer CM, Bourque JM. Coronary microvascular dysfunction, microvascular angina, and treatment strategies. JACC. 2015;8:210-20.

8. Thomson LE, Wei J, Agarwal M, et al. Cardiac magnetic resonance myocardial perfusion reserve index is reduced in women with coronary microvascular dysfunction. A National Heart, Lung, and Blood Institute-sponsored study from the Women's ischemia syndrome evaluation. Circulation. 2015;8:e02481.

9. Wu CC, Feldman MD, Mills JD, et al. Myocardial contrast echocardiography can be used to quantify intramyocardial blood volume: New insights into structural mechanisms of coronary autoregulation. Circulation. 1997;96(3):1001-11.

10. Schindler TH, Schelbert HR, Quercioli A, Dilsizian V. Cardiac PET imaging for the detection and monitoring of coronary artery disease and microvascular health. JACC. 2010;3(6):623-40.

11. Yong AS, Layland J, Fearon WF, et al. Calculation of the index of microcirculatory resistance without coronary wedge pressure measurement in the presence of epicardial stenosis. JACC. 2013;6:53-8.

12. Mohy-Ud-Din H, Lodge MA, Rahmim A. Quantitative myocardial perfusion PET parametric imaging at the voxel-level. Phys Med Biol. 2015;60(15):6013-37.
13. Kanatsuka H, Lamping KG, Eastham CL, Marcus ML. Heterogeneous changes in epimyocardial microvascular size during graded coronary stenosis. Evidence of the microvascular site for autoregulation. Circ Res. 1990;66(2):389-96.

14. Wu XS, Ewert DL, Liu YH, Ritman EL. In vivo relation of intramyocardial blood volume to myocardial perfusion. Evidence supporting microvascular site for autoregulation. Circulation. 1992;85(2):730-7.

15. Yang J, Huang S, Mega M, et al. Investigation of partial volume correction methods for brain FDG-PET studies. IEEE Trans Nucl Sci. 1996;43:3322-7.

16. Chan C, Liu H, Grobshtein Y, Stacy MR, Sinusas AJ, Liu C. Noise suppressed partial volume correction for cardiac SPECT/CT. Med Phys. 2016;43(9):5225.

17. Erlandsson K, Buvat I, Pretorius PH, Thomas BA, Hutton BF. A review of partial volume correction techniques for emission tomography and their applications in neurology, cardiology and oncology. Phys Med Biol. 2012;57(21):R119-59.

18. Liu H, Chan C, Grobshtein Y, et al. Anatomical-based partial volume correction for low-dose dedicated cardiac SPECT/CT. Phys Med Biol. 2015;60(17):6751-73.

19. Mohammed SF, Hussain S, Mirzoyev SA, Edwards WD, Maleszewski JJ, Redfield MM. Coronary microvascular rarefaction and myocardial fibrosis in heart failure with preserved ejection fraction. Circulation. 2015;131(6):550-9.

20. Porter TR. Capillary blood flow abnormalities in the skeletal muscle and microvascular complications in diabetes lessons that cannot be learned from larger vessels. J Am Coll Cardiol. 2009;53(23):2184-5.

21. Behrenbeck TR, McCollough CH, Miller WL, et al. Early changes in myocardial microcirculation in asymptomatic hypercholesterolemic subjects: As detected by perfusion CT. Ann Biomed Eng. 2014;42(3):515-25.

22. Miller WL, Behrenbeck TR, McCollough $\mathrm{CH}$, et al. Coronary microcirculation changes in non-ischemic dilated cardiomyopathy identified by novel perfusion CT. Int $\mathrm{J}$ Cardiovasc Imaging. 2015;31(4):881-8. 